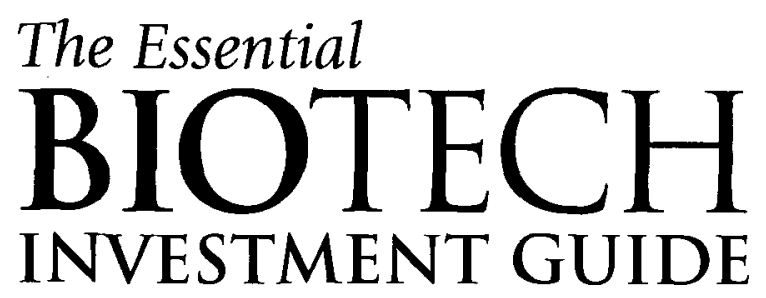


This page is intentionally left blank 


\section{The Essential}
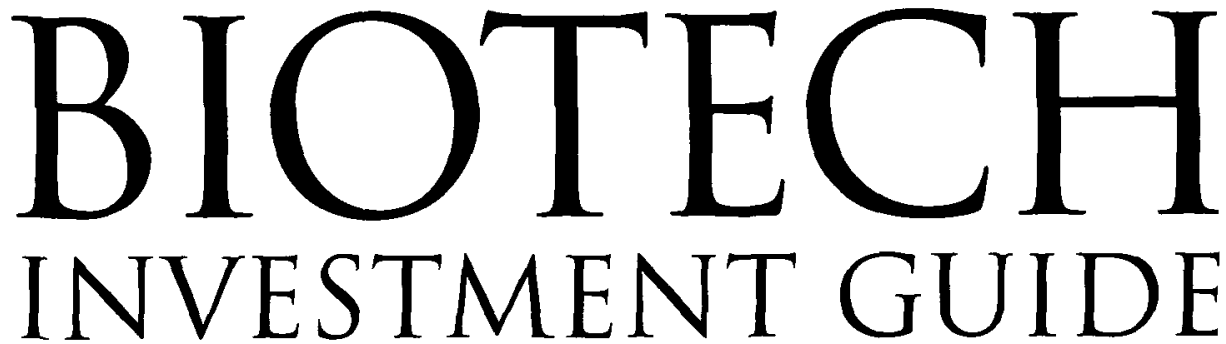

\section{How to Invest in the Healthcare Biotechnology \& Life Sciences Sector}

Chilung Mark Tang, Ph.D. World Technology Investment Group Corporation, USA 
Published by

World Scientific Publishing Co. Pte. Ltd.

P O Box 128, Farrer Road, Singapore 912805

USA office: Suite 202, 1060 Main Street, River Edge, NJ 07661

UK office: 57 Shelton Street, Covent Garden, London WC2H 9HE

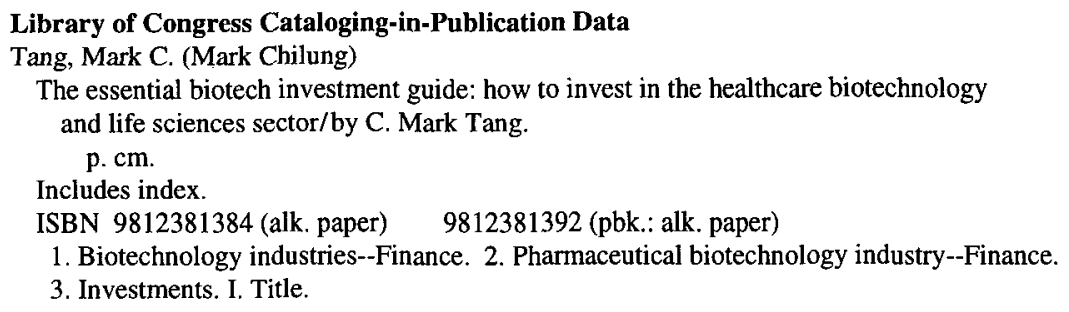

HD9999.B442 T36 2002

$332.63^{\prime} 2--\mathrm{dc} 21$

2002033070

\section{British Library Cataloging-in-Publication Data}

A catalogue record for this book is available from the British Library.

\section{Copyright $\odot 2002$ by C. Mark Tang, Ph.D.}

All rights reserved. This book, or parts thereof, may not be reproduced in any form or by any means, electronic or mechanical, including photocopying, recording or any information storage and retrieval system now known or to be invented, without written permission from the Author.

Requests for permission or further information should be addressed to the:

Permission Department, World Scientific Publishing Co.Pte Ltd., 5 Toh Tuck Link, Singapore 596224.

Disclaimer. The materials and opinions herein are based upon publicly available information believed to be reliable, and may change without notice. The author or Publisher shall not in any way be liable for claims relating to them, and makes no express or implied representations or warranties as to their accuracy or completeness or for statements or errors contained in, or omissions from, them. The information and analyses contained herein are not intended as tax, legal or investment advice and may not be suitable for your specific circumstances; accordingly, you should consult your own tax, legal, investment or other advisors to determine such suitability. Any investment returns, past, hypothetical or otherwise, are not indicative of future performance. 
This book is dedicated to the Tang Family

\author{
Gloria \\ Alexander \\ Sharon
}


This page is intentionally left blank 


\section{Preface}

I became interested in investing in biotech in the late 80 s while I was a $\mathrm{Ph} . \mathrm{D}$. candidate in the Biochemistry Department at the University of California, Riverside, in an adjunct molecular endocrinology laboratory of UCLA School of Medicine. After I finished the doctoral degree, I moved to New York in 1993 and worked at the prestigious Rockefeller University. I was a founder and publisher of Bio/Medical Technology Stock newsletter in 1993. The letter was later acquired in 1996 when I became a biopharmaceutical investment banker/venture capitalist. During 1993-1996, I read as many books as I could find on investing and valuation. I became interested in value investing by Graham and Buffett. Later, I was able to take finance classes in the part-time MBA program at New York University and finally obtained an MBA in finance. In 1997, suggested by Michael Penn, I decided to write a handbook on investing in biotech. Since then, through Mr. Market's many ups and downs, and my working at different capacities on Wall Street, I finally finished the assignment in late 2001.

I believe this investment guide is unique in that it aims to have both breadth and depth. I also endeavored to discuss biotech investing from asset management and risk management point of, in the hope that it will be useful for biotech experts, bioentrepreneurs, and individual biotech investors alike. 
This page is intentionally left blank 


\section{Acknowledgements}

The author is grateful to the following individuals for their comments and editorial and proofreading assistance:

Melinda Mingus, M.D. (an NYU Stern Business School alumna), Pascal Moulin, Calorina Morrillas, Cortney Williams, Fran Bartlett, Mary Miller, Liz Weston, Don Cooperman, Esq, of Cooperman and Co. (Chapter 14), Y. Zhu, M.D. Ph.D., of Cornell Medical College (Chapter 2), Z. Yang, Ph.D. (Chapter 2), and Jonathan Lin of Citigroup (Appendix A). 
This page is intentionally left blank 


\section{Contents}

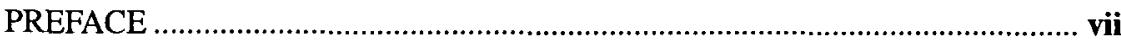

ACKNOWLEDGEMENTS ....................................................................... ix

FIGURES AND TABLES ............................................................................. xviii

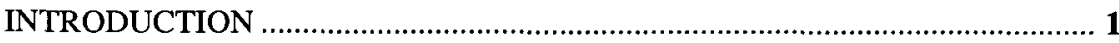

Biotech Stock For Wealth Growth: An Essential Portion Of Asset

ALLOCATION .................................................................................................. 1

Biotechnological Innovation Benefits Society ................................................ I

Biotech Sector Outperforms .......................................................................... 1

Strong Fundamentals and Product Pipelines .................................................... 2

Strong Growth of Revenue and Profits .............................................................. 6

Biotechnology Differs from the Internet ............................................................ 6

Stock is Essential for Wealth Growth ................................................................. 7

Stock for Outpacing Inflation .......................................................................... 11

Why Biotech? ............................................................................................. 13

Positive Demography: Our Baby Boomer Population ………….................... 15

Positive Demographics Trend .................................................................... 16

Positive Technology Trend ........................................................................... 16

Big Pharma and the Biotech Industry-Complementary to Each Other.......... 16

Supply Demand Imbalance? ...................................................................... 20

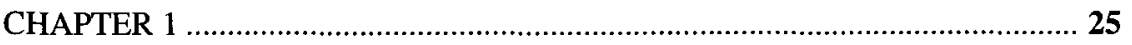

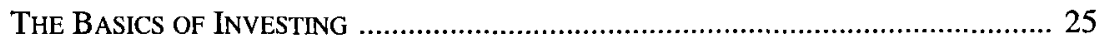

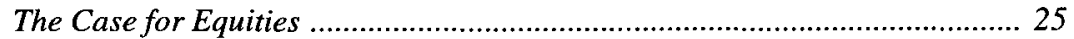

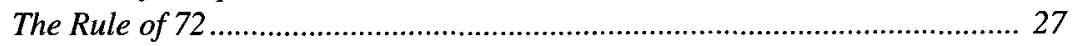

The Power of Compounding ....................................................................... 28 
Opportunity Cost ...................................................................................... 29

Relationship Between Risk and Return .................................................. 29

Dollar-Cost Averaging ............................................................................ 29

Purchasing Power and Inflation-Is a CD Really Safe? ............................ 31

Holding Period and Risk ........................................................................ 32

Understanding Risks ........................................................................... 33

Diversification-Minimizing Your Investment Risk ................................... 35

Past Reasons for Not Investing ................................................................ 38

Historic Return and How Much Return One Should Expect.......................... 39

Technical Analysis ...................................................................................... 39

Investment Vehicles Available to Achieve Your Goals .................................. 40

Vehicle One: Stocks .............................................................................. 40

Vehicle Two: Bonds.................................................................................. 44

Vehicle Three: Cash .................................................................................. 45

Vehicle Four: Mutual Funds.................................................................... 45

Vehicle Five: Unit Investment Trust ........................................................ 46

Vehicle Six: Managed Accounts ............................................................. 47

Vehicle Seven: Alternative Investment ..................................................... 47

Asset Allocation ....................................................................................... 47

Managing Your Portfolio and Wealth ...................................................... 48

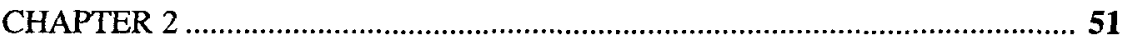

AN INTRODUCTION TO BIOTECHNOLOGY AND GENOMICS .................................. 51

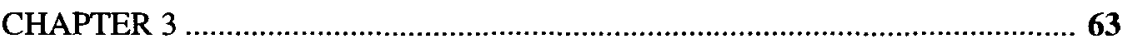

UNDERSTANDING BIOTECHNOLOGY INVENTION AND THE FDA APPROVAL

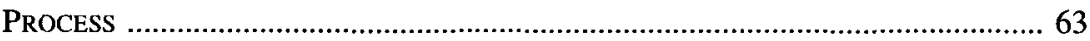

Part I: Biotechnology Inventions and Patents .......................................6.63

Part II: The FDA Approval Process for Drugs .......................................... 67

Stages of the FDA Approval Process............................................................. 67

Drug Review Glossary .......................................................................... 75

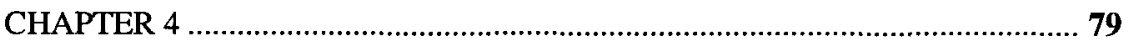

INTRODUCTION TO BIOTECH INVESTING .................................................... 79

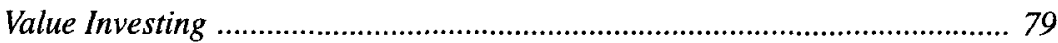

Growth Investing ................................................................................. 79

The Industry Life Cycle ................................................................... 80

Characteristics of Investing in Biotech ................................................ 81

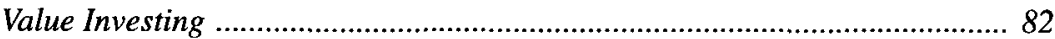

Biotechnology Investment Trading Rules ........................................ 86

Winning Biotechnology Company Attribute List ...................................... 89 


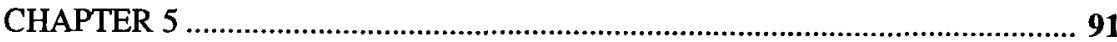

How to Value AND InVEST In A BIOTECH CoMPANY ...................................... 91

Step One: The Economic and Business Cycle ............................................. 93

Step Two: Industry Sector Analysis ........................................................... 95

Biotech Industry Sector Analysis ............................................................. 95

Step Three: Biotech Company Analysis ................................................... 97

Valuation: Should I Buy This Biotech Stock Now? ....................................... 98

Method 1: Relative Valuation ................................................................... 100

Comparisons of P/E Ratios ...................................................................... 102

Method 2: Discounted Cash Flow (DCF) Analysis ...................................... 116

Method 3: Sum-of-the-Parts Valuations ....................................................... 116

Method 4: Dividend Discount Model (DDM) ............................................ 119

Method 5: Subscriber-Based Valuations ........................................................ 119

Method 6: Economic Value Added (EVA) ................................................... 120

Method 7: Yield-Based Valuations-Valuing Market as an Example ........... 120

Qualitative Component of the Valuation ................................................... 120

Risks of Biotech Companies ................................................................. 120

Summary: Which Model Is Best for Valuing Biotech Companies? ............. 121

Part II: Investing in Undervalued Healthcare Biotech-Proactive

Value Investing in Healthcare ${ }^{T M}$................................................... 123

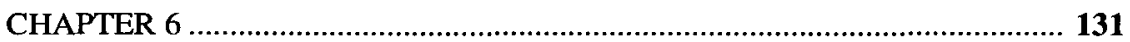

INVESTING IN BIOTECHNOLOGY MutUAL FundS ......................................... 131

Don't Put All Your Eggs in One Basket .................................................. 131

Purchasing Power ........................................................................ 132

Professional Management ................................................................... 133

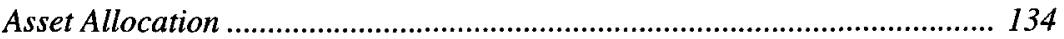

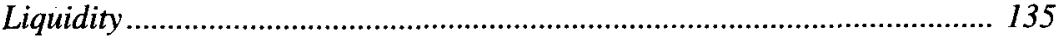

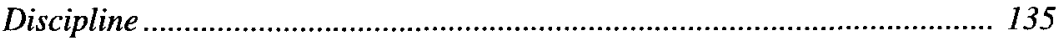

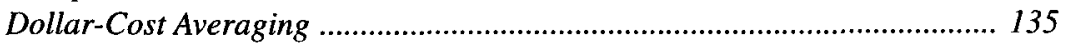

The Case for Index Funds .................................................................... 136

Why Stock or Stock Mutual Funds? ........................................................ 136

The Cost of Delaying .................................................................................. 139

The Benefits of Investing in Biotech Mutual Funds .................................. 139

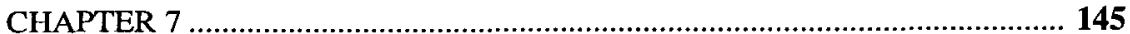

HealthCare Biotech Index Investing: Strategies Using Exchange-

TRADED-Funds (ETFs), BIOTECH ISHARES, AND BOXES ........................... 145

The Case for Index Funds .................................................................. 145

I. Exchange-Traded Funds (ETFs) ................................................. 146 
Advantages of ETFs..................................................................... 147

Options Strategies for Suitable Clients ................................................... 149

Index-Linked ETFs .................................................................................... 150

II. Index Investing ................................................................................. 151

III. Strategies Using Biotech and Pharmaceutical BOXES ...................... 152

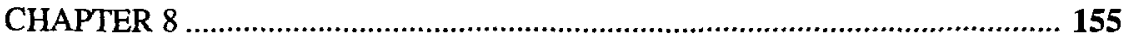

RisK MANAGEMENT CONSIDERATIONS FOR BIOTECH INVESTORS WITH

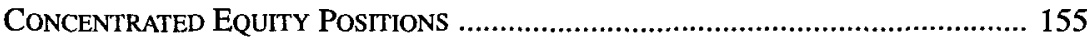

Alternative 1: Retention of the Position and Associated Risks .................. 156

Alternative 2: Liquidation of the Position and Reinvestment of

Net Proceeds ........................................................................................ 158

Alternative 3: Hedging, Monetizing, and Diversification Strategies .......... 158

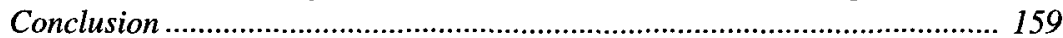

Glossary .................................................................................. 162

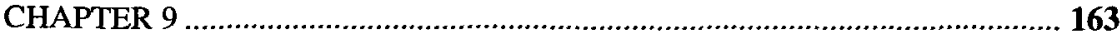

Managing Biotech Stock Options: Your EMPLoyee Benefits ................... 163

Non-Qualified Biotech Options ........................................................... 165

Incentive Biotech Options ..................................................................... 166

Your Biotech Option Plan ..................................................................... 167

Alternative Biotech Stock Acquisition Programs ................................. 170

83 (b) Election ........................................................................ 171

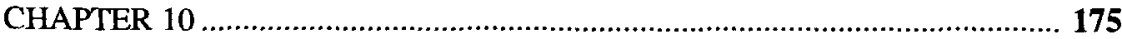

An Introduction to HealthCare Biotechnology Hedge

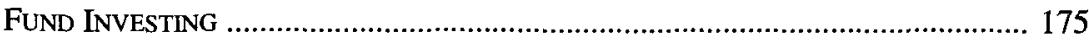

Hedge Fund Investment Styles ........................................................... 176

Three Ways to Participate Healthcare-Biotech Hedge Funds .................. 177

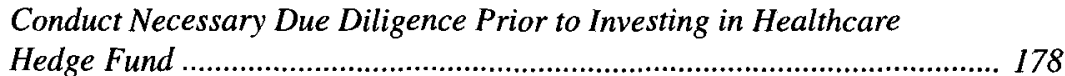

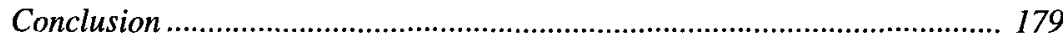

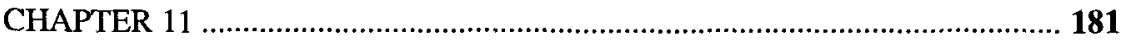

An Introduction to HealthCare Biotechnology Private Equity

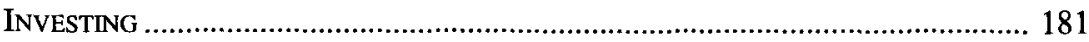

I. Healthcare Venture Capital .............................................................. 182

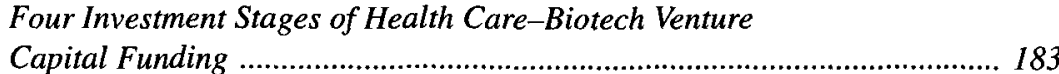

II. Healthcare Buyout Funds ....................................................... 183 
III. Special Situations in Healthcare ..................................................... 184

Key Considerations ................................................................................ 184

How to Participate in Private Equity Investing .......................................... 185

Five Basic Ways To Participate in Healthcare Private Equity Investing ...... 186

Measuring Performance of Healthcare Biotech Private Equity Funds ........ 187

Conclusion ..................................................................................... 188

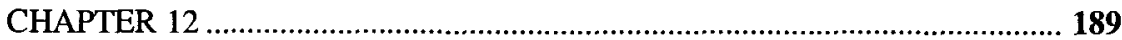

Retirement Planning Considerations For Biotech Executives and

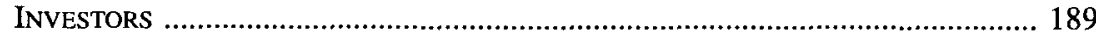

Changing Careers: Affecting Your Retirement Savings ............................ 189

Direct And Indirect Rollovers: Spinning Your IRA ................................... 190

The Tax-Deferred Rollover ................................................................ 192

Retirement: Strategy ....................................................................... 192

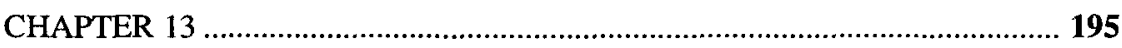

Charitable Disposition of APPRECiATEd Biotech Stocks ............................ 195

I. Private Foundations ........................................................................ 196

II. Charitable Remainder Trusts ......................................................... 197

Conclusion ........................................................................................ 198

III. Alternative Charitable Gift Program Through National

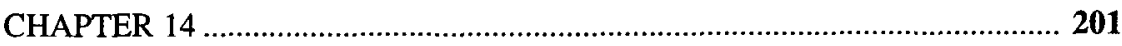

Managing Your Biotech Wealth-Estate Planning for Biotech

INVESTORS, EXECUTIVES, AND FouNDERS .................................................. 201

Other Trust Strategies to Minimize Estate Taxes ....................................... 208

Conclusion .......................................................................................... 213

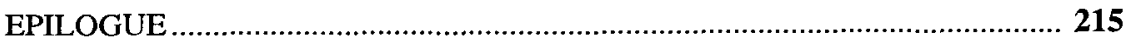

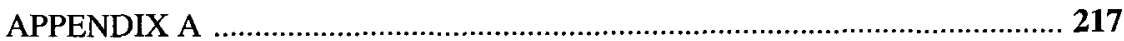

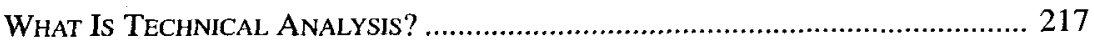

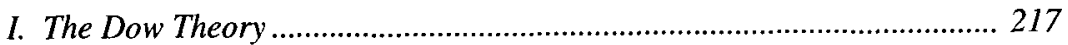

II. Four Groups of Technical Indicators ............................................. 218

III. Top-Down Technical Analysis ..................................................... 222

IV. Reading Chart Patterns.................................................................. 222

Patterns Indicating a Reversal in a Stock Price Trend ............................. 224

Patterns Indicating a Consolidation in a Stock Price Trend ........................ 224 


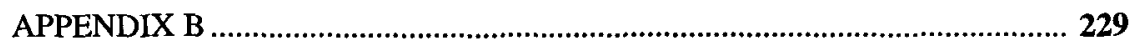

BIOTECH AND LIFE SCIENCE GLOSSARY ................................................... 229

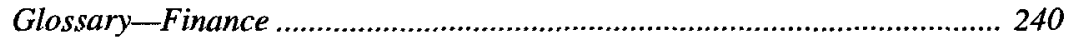

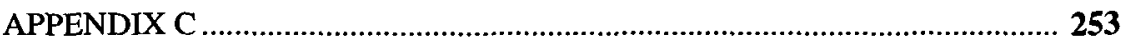

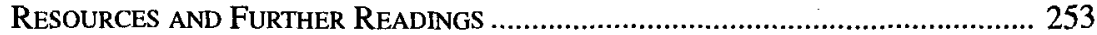

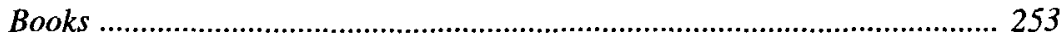

Web Sites ............................................................................................ 254

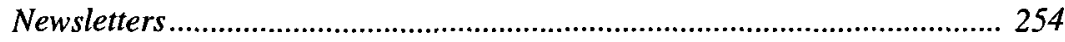

Newspapers and Magazines ................................................................ 254

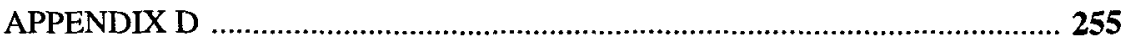

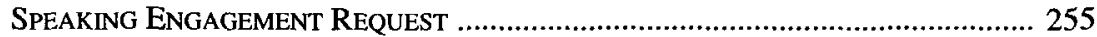

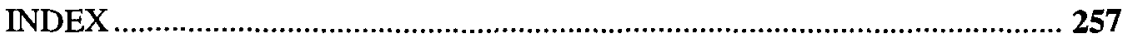




\section{Figures And Tables}

Figure 1-1. Performance of BTK, SP, Internet, and NASDAQ over the last 3 and 10 Years

Table 1-1. Selected Profitable Biotech Valuation Comparisons (as of 12/31/01)

Table 1-2. Selected Major Pharmaceuticals Valuation Comparisons (as of 12/31/01)

Table 1-3. Selected 2001 Biologic Drug Approvals ................................................ 8

Table I-4. Selected FDA Approved Biopharmaceutical Drugs ............................ 9

Table 1-5. Historic Returns for Different Asset Class ........................................... 11

Table I-6. Effect of Inflation on Investment Growth........................................... 11

Figure I-2. Hypothetical Growth of a \$1 Investment in Four Traditional

Asset Classes.................................................................................................. 12

Table I-7. The "Graying" of America: Americans Over the Age of 65 ............... 17

Table I-8. Annual Pharmaceutical Expenditures by Age Group .......................... 17

Table I-9. Drug Patent Expiration Table ........................................................ 18

Table I-10. Biotech Added Value for Big Pharma-Biotech Covers

Technologies Outside of Big Pharmaceuticals ...................................................... 20

Figure I-3. Patents Granted in the U.S. Rose to Record Highs in the

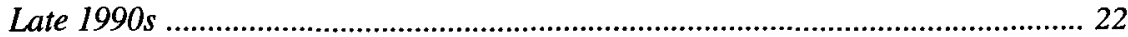

Table I-1I. The 20th Century's Greatest Engineering Achievements................... 23

Table I-12. The Leading Biotech Drugs .......................................................... 24

Figure 1-1. Over Time Equity Outperforms Other Asset Classes ......................... 26

Table 1-1. Average 20-Year Return from 1981-2001 .......................................... 27

Table 1-2. Number of Years Needed to Double Your Investment ........................... 27

Table 1-3. The Power of Compounding and Cost of Waiting ............................... 28

Figure 1-2. The Power of Compounding-Hypothetical Investment in Stocks ....... 30

Figure 1-3. The Relationship Between Risk and Return ................................... 30 
Table 1-4. An Example of Dollar-Cost Averaging ............................................ 31

Table 1-5. Annual Average Inflation From 1925 to 1999 .................................... 31

Table 1-6. How Much Money You Need to Keep \$100 Dollars

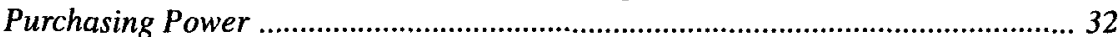

Table 1-7. Historic Returns of Cash and Equivalent After Inflation and

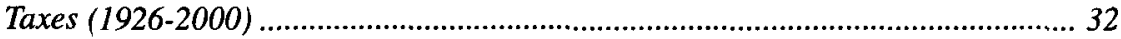

Table 1-8. Holding Period and Risk ............................................................... 33

Figure 1-4. Holding Period and Return ........................................................ 34

Figure 1-5. Investment Pyramid-Relationship Between Risk and Return ............ 34

Figure 1-6. Asset Allocation Among Classes .................................................... 36

Figure 1-7. Diversification Among Stock, Bond and Money Markets ................... 36

Figure 1-8. Diversification Among Bonds ..................................................... 37

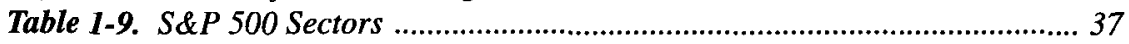

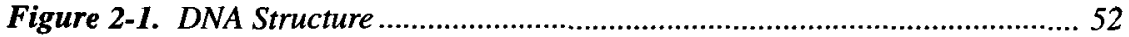

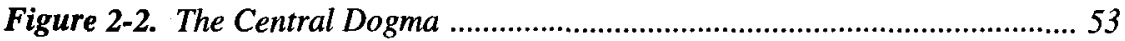

Figure 2-3. Protein Translation ................................................................. 55

Figure 2-4. Growth of Biological Data ............................................................. 57

Table 2-1. Selected Biotech Stocks and Their Symbols .................................... 58

Table 2-2. Biotech Companies by Type of Technologies or Applications .............. 60

Figure 2-5. Bio-Technology Chart ....................................................................62 62

Figure 3-1. Stages of the FDA Approval Process ...............................................69 69

Table 3-1. FDA Review Times .......................................................................... 72

Figure 3-2. Risk Data Analysis of Drug Development and Approval ................... 73

Table 3-2. Risk of Clinical Trials ....................................................................... 74

Table 4-1. Typical Industry Life Cycle ............................................................. 81

Figure 4-1. Drug Development Risk ............................................................ 90

Table 5-I. Summary of General Valuation Methods ........................................... 92

Table 5-2. Classic Business Cycle Model ......................................................... 94

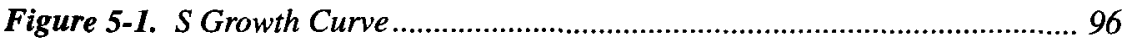

Table 5-3. PEG Ratios of Big Pharmaceutical Companies in North America .... 105

Table 5-4. PEG Ratios of Profitable Biopharmaceutical Company in the U.S. .. 106

Table 5-5. PEG Ratios of Non-north American Pharmaceutical Companies ...... 107

Table 5-6. Comparative Analysis-IPO ........................................................ 111

Table 5-7. Comparative Analysis-Genomic Company ....................................112

Table 5-8. Estimating Net Present Value (Intrinsic Value) of a

Biotech Company ........................................................................................... 117

Table 5-9. Estimate of Intrinsic Value of Five Major Profitable

Biotech Company ............................................................................................ 118

Table 5-10. Sum of Parts Valuation of Drug Candidate Pipelines ...................... 119

Table 5-11. Business Model and Valuation Differences between Pharma and

Biotech. 
Table 5-12 Pros and Cons of Valuation Techniques.......................................... 122

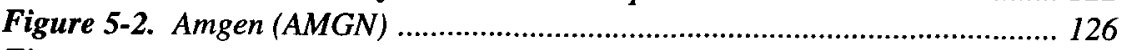

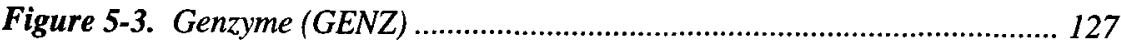

Figure 5-4. Chiron (CHIR) ................................................................... 127

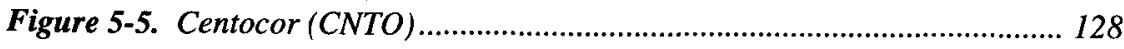

Figure 5-6. Cephalon (CEPH) ................................................................ 128

Figure 5-7. Human Genome Sciences (HGSI) ................................................. 129

Figure 5-8. Immunex (IMNX) .................................................................. 129

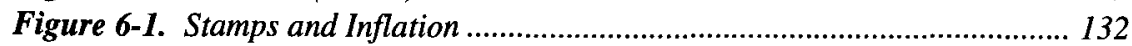

Figure 6-2. Risk-Reward Parameter/Pyramid .............................................. 133

Table 6-1. The Value of Professional Management in Biotechnology-10-Year

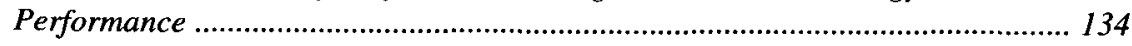

Figure 6-3. Value Versus Growth ............................................................ 138

Figure 6-4. Positive Investment Return and Holding Period ............................ 142

Figure 6-5. Cost of Missing Best Months (1970-2001) ................................. 142

Table 6-2. Healthcare-Biotechnology Funds .................................................. 143

Figure 7-1. Institutional Strategy Evolves to Core/Satellite ............................ 148

Figure 7-2. Tax Strategy: Loss Harvesting .................................................. 154

Table 7-1. How Do Pharmaceutical and Biotech BOXES Work? ........................ 154

Figure 8-1. Systematic Risk and Company-Spcific Risk .................................... 156

Figure 8-2. Risk Management Strategies for Low-cost, Concentrated and/or Restricted Biotech Stock ............................................................................... 157

Table 8-1. Hedging and Monetizing Strategies ............................................. 161

Table 8-2. Risk Management-Summary of Strategic Outcomes........................ 161

Table 9-1. How Do Biotech Options Work? ................................................... 167

Table 9-2. Biotech Stock Options Vesting Methods .......................................... 168

Table 11-1. The Pros and Cons of Private Equity Investing ......................... 182

Figure 12-1. Retirement Sources ................................................................. 191

Figure 12-2. Tax Deferring Advantage ....................................................... 193

Table 13-1. Tax Benefit of Depositing Appreciated Biotech Stocks

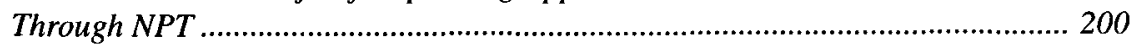

Table 14-1. Comparison of Four Alternatives .................................................. 204

Figure 14-1. Tax Saving through Estate Planning ...................................... 214

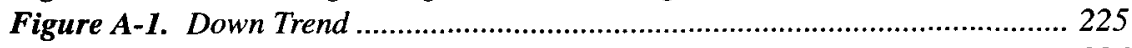

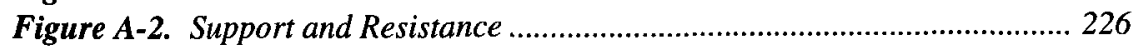

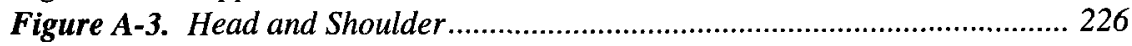

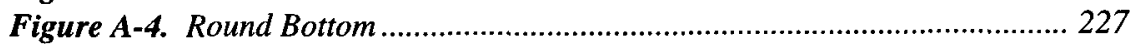

Figure A-5. Double Bottom ................................................................... 227

Figure A-2 to A-5. Chart Patterns .............................................................. 227 